\title{
Patient preferences for treatment of metastatic melanoma
}

\author{
Carol Mansfield*,1, Briana Ndife ${ }^{2}$, Joyce Chen ${ }^{2}$, Kathleen Gallaher ${ }^{1}$ \& Sameer Ghate ${ }^{2}$ \\ ${ }^{1}$ RTI Health Solutions, Research Triangle Park, NC 27709, USA \\ ${ }^{2}$ Novartis Pharmaceuticals Corporation, East Hanover, NJ 07936, USA \\ *Author for correspondence: Tel.: +1 919541 8053; carolm@rti.org
}

\begin{abstract}
Aim: To investigate patient preferences for clinical attributes of first-line metastatic melanoma treatments. Materials \& methods: A discrete-choice experiment and best-worst scaling exercise were used to assess relative preferences for treatment attributes. Results: The 200 survey respondents had distinct preferences. Avoiding a $30 \%$ risk of colitis or hormone gland problems and avoiding severe fever were more important to respondents than avoiding a $20 \%$ risk of extreme sun sensitivity $(p<0.05)$. Patients preferred taking pills to receiving intravenous infusions in a clinic. When attributes were combined, approximately $85 \%$ of respondents preferred a risk profile similar to targeted therapy over a profile similar to immunotherapy, holding efficacy constant. Conclusion: Taking patient preferences into account can help patients get the full benefit from metastatic melanoma therapies.
\end{abstract}

First draft submitted: 21 November 2018; Accepted for publication: 14 January 2019; Published online: 29 January 2019

Keywords: adverse effects - B-RAF $\bullet$ drug therapy $\bullet$ immunotherapy $\bullet$ melanoma $\bullet$ patient preference $\bullet$ progression-free survival $\bullet$ targeted therapy

The introduction of targeted therapy and immune checkpoint inhibitors for metastatic melanoma has provided patients and physicians with unprecedented treatment options. In particular, patients whose tumors express mutant forms of the BRAFV600 gene can choose from different agents that target their tumor's gene expression profile (i.e., targeted therapy) or immune checkpoint inhibitors that block certain proteins in the immune system and help restore T-cell function. The first available immune checkpoint inhibitor, ipilimumab (Bristol-Myers Squibb, NY, USA), introduced in 2011, was joined by targeted BRAF/MEK inhibitors such as dabrafenib and trametinib (Novartis Pharmaceuticals Corporation, Basel, Switzerland), cobimetinib and vemurafenib (Genentech, Inc. CA, USA), and additional immune checkpoint inhibitor agents nivolumab (Bristol-Myers Squibb) and pembrolizumab (Merck \& Co., Inc., NJ, USA) [1]. Each of these treatment options presents trade-offs in terms of efficacy, adverse event (AE) profiles and administration mode and frequency [1,2]. Taken together, the choices now available to BRAFV600-positive metastatic melanoma patients present a complex set of decisions that must be weighed by physicians and patients as they choose a treatment regimen [3-9].

The American Society of Clinical Oncology recently issued consensus guidelines on physician-patient communication that stress physicians' responsibility to consider patient's goals for care [10]. Further, the US FDA's ongoing initiative aimed at patient-centered care is expected to increase the weight placed on patient preferences in regulatory decision making [11]. An FDA guidance document on incorporating patient preferences has been issued [12], and the agency has committed to increasing the patient voice in regulatory decision making.

As focus on the patient experience of treatment increases, a need arises for studies that address patient preferences in a systematic, reproducible way. One such method, the discrete-choice experiment (DCE), has emerged as an accepted methodology for gathering and analyzing quantitative data on the preferences of patients and caregivers in the healthcare setting [13]. Findings generated through DCE experiments have begun to be used to help guide regulatory decision making [14] and the development of tools that support shared decision making between patient and caregiver [15].

In the realm of metastatic melanoma care, few studies have been conducted to assess patient preferences in the targeted therapy era. Liu et al. conducted two studies that examined treatment preferences of patients with advanced

Future 8 Medicine 
melanoma along with oncology nurses [16] and oncologists [17]. These studies revealed that patients placed more importance on overall survival (OS) compared with physicians [17]. Nurses and patients held similar views, with OS and reducing the risk of AEs being most important [16]. However, multiple outcome measures were used in these studies, and the risk of any serious AEs was presented as a single attribute. Among available treatments, serious $\mathrm{AEs}$ vary, and the risk of any single $\mathrm{AE}$ varies with individual medication or combination therapy [3,18]. Further, as these treatments and novel combination therapies are evolving, limited real-world data on OS and several of the other outcome measures are consistently available across existing treatments. To further elaborate the clinical attributes most important to advanced or metastatic melanoma patients, we designed a patient preference study that incorporated real-world clinical attributes among currently available first-line treatments.

\section{Materials \& methods}

\section{Study design}

We conducted a cross-sectional study incorporating two types of patient preference methods, a DCE and a best-worst scaling (BWS) exercise.

The study examined the following end points:

- Patient preferences for metastatic melanoma treatments (overall, among respondents with an initial diagnosis of stage IV disease vs those with an earlier disease stage at diagnosis, and among respondents aged 47 years and older vs those younger than 47 years), given a choice among hypothetical treatment choices drawn from known clinical attributes of treatments approved for patients whose tumors express mutant BRAFV 600 protein, using a DCE exercise;

- Patient preferences for mode and frequency of treatment administration, using a BWS exercise.

\section{Study population}

Global Perspectives (Norwich, UK) recruited a convenience sample through patient associations, proprietary databases and opt-in online panels. Potential respondents were sent an email with a unique link to the survey. The first few questions in the survey screened potential respondents for eligibility.

To gain access to the survey, participants had to be 18 years or older, have a self-reported physician diagnosis of advanced or metastatic melanoma defined as diagnosis of stage III or stage IV melanoma or the melanoma had spread to other parts of the body since diagnosis, be able to read and understand English, and provide informed consent to participate in the survey. Respondents were compensated US $\$ 75$.

\section{Survey instrument}

Discrete-choice experiment

We developed and administered a DCE survey following guidelines for good research practices [19]. The survey methodology elicits patient preferences for clinical attributes that differ among treatments. Survey participants choose from a series of two presented hypothetical treatment profiles that combine attribute levels. Profiles are paired following a defined experimental design.

For this study, the DCE survey instrument was developed to elicit the relative importance of metastatic melanoma treatment attributes, using validated methods to measure preferences [20]. This study evaluated six attributes defined by three levels (Table 1). We chose the attributes based on available clinical literature for immunotherapies and targeted therapies used to treat BRAFV600-positive metastatic melanoma. The levels of the attributes were selected to span the range of outcomes observed in clinical data from previous studies with individual and combined therapies [21-31]. On the basis of limited availability of data on OS in some of the comparator treatments, we chose to present survey respondents with progression-free survival (PFS) data to best represent reported clinical outcomes [21-31]. For AEs, fever and the risk of extreme sun sensitivity were selected as representative of targeted therapies, and risk of colitis and hormone gland problems were selected as representative of immune checkpoint inhibitors. Oral administration was selected as representative for targeted therapies, and intravenous (IV) infusion was selected as representative for immune checkpoint inhibitors. The relevance of the attributes and levels was reviewed by clinical experts. The descriptions and levels were evaluated in 12 pretests with patients having a selfreported physician diagnosis of advanced or metastatic melanoma. Changes to the survey instrument were made based on participant feedback. 


\begin{tabular}{|c|c|}
\hline Discrete-choice experiment attributes & Levels \\
\hline How long the medicine will keep the cancer stable (PFS) & $\begin{array}{l}13 \text { months } \\
7 \text { months } \\
4 \text { months }\end{array}$ \\
\hline How you take the medicine & $\begin{array}{l}\text { Pills taken twice-a-day at home } \\
\text { IV infusion for } 30 \text { minutes every } 3 \text { weeks in the doctor's office } \\
\text { IV infusion for } 2.5 \text { hours every } 3 \text { weeks in the doctor's office for four doses, then IV } \\
\text { infusion for } 60 \text { minutes every } 2 \text { weeks } \ddagger\end{array}$ \\
\hline Fever for a few days each month ${ }^{\dagger}$ & $\begin{array}{l}\text { None } \\
\text { Mild to moderate } \\
\text { Severe }\end{array}$ \\
\hline Risk of tears or holes in your intestines (colitis) ${ }^{\ddagger}$ & $\begin{array}{l}0 \% \\
10 \% \\
30 \%\end{array}$ \\
\hline Risk of hormone gland problems ${ }^{\ddagger}$ & $\begin{array}{l}0 \% \\
10 \% \\
30 \%\end{array}$ \\
\hline Risk of extreme sensitivity to the sun ${ }^{\dagger}$ & $\begin{array}{l}0 \% \\
4 \% \\
20 \%\end{array}$ \\
\hline \multicolumn{2}{|l|}{ Best-worst scaling items } \\
\hline \multicolumn{2}{|l|}{ One pill once-a-day at home ${ }^{\dagger}$} \\
\hline \multicolumn{2}{|l|}{ Two pills twice-a-day at home ${ }^{\dagger}$} \\
\hline \multicolumn{2}{|l|}{ Four pills twice-a-day at home ${ }^{\dagger}$} \\
\hline \multicolumn{2}{|c|}{ Two pills twice-a-day, plus one additional pill once-a-day at home ${ }^{\dagger}$} \\
\hline \multicolumn{2}{|l|}{ Three pills once-a-day for 21 days, then stop for 7 days (repeat) ${ }^{\dagger}$} \\
\hline \multicolumn{2}{|c|}{ Three pills once-a-day for 21 days, then stop for 7 days (repeat), plus four pills twice-a-day at home ${ }^{\dagger}$} \\
\hline \multicolumn{2}{|l|}{ IV for 30 minutes every 3 weeks in the doctor's office ${ }^{\ddagger}$} \\
\hline \multicolumn{2}{|l|}{ IV for 60 minutes every 2 weeks in the doctor's office ${ }^{\ddagger}$} \\
\hline \multicolumn{2}{|c|}{ IV for 2.5 hours every 3 weeks for four doses, then IV for 60 minutes every 2 weeks in the doctor's office $\ddagger$} \\
\hline $\begin{array}{l}\dagger \text { Associated with targeted therapies. } \\
\text { ¥Associated with immunotherapies. } \\
\text { IV: Intravenous; PFS: Progression-free survival. }\end{array}$ & \\
\hline
\end{tabular}

The experimental design was created in Sawtooth Software (WA, USA) using a D-efficient algorithm to construct a fractional factorial experimental design [32-35]. The design was evaluated for level balance and correlation. The full design included 27 choice questions that were divided into three blocks of nine questions. Each respondent was randomly assigned to one block of nine DCE questions. Figure 1A presents an example DCE choice question.

\section{Best-worst scaling}

To further elucidate relative patient preferences for modes of administration, we developed a BWS study, which permits a ranking of the perceived burden of all items included in the exercise and a measure of relative magnitude of the burden of each item [36]. The study was designed using object-case BWS [37,38], which has been previously applied in healthcare settings [36,39-42]. The BWS included nine different modes and schedules of administration based on existing targeted therapy and immunotherapy. Table 1 presents the BWS item levels evaluated in the study.

The BWS questions were created from an experimental design created in Sawtooth Software with one block of 12 questions. Each respondent was shown the same 12 questions in a random order. Each question contained a set of three items and asked to identify the highest- and lowest-ranked item in the set. Figure 1B presents an example BWS question.

\section{Statistical analyses \\ Discrete-choice experiment}

The DCE treatment choice questions were analyzed using a random-parameters logit (RPL) model estimated using NLOGIT version 5.0 (Econometric Software, Inc., NY, USA), which accounts for variations among individual 


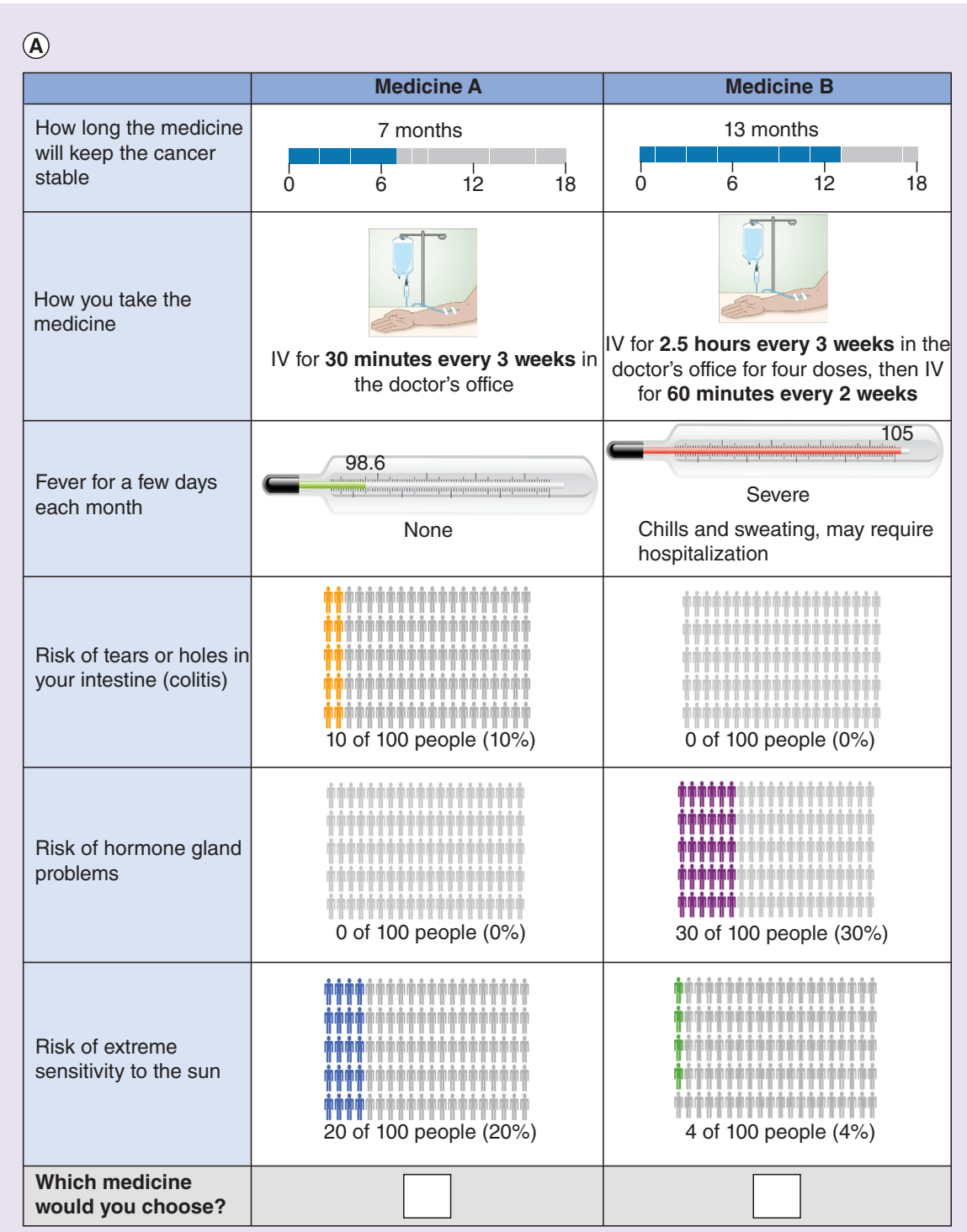

(B)

\begin{tabular}{|c|c|c|}
\hline $\begin{array}{c}\text { I would prefer } \\
\text { this } \\
\text { the most } \\
\text { (check only one) }\end{array}$ & Treatment features & $\begin{array}{c}\text { I would prefer } \\
\text { this } \\
\text { the least } \\
\text { (check only one) }\end{array}$ \\
\hline & $\begin{array}{l}\text { IV for } 2.5 \text { hours every } 3 \text { weeks for } \\
4 \text { doses, then IV for } 60 \text { minutes every } \\
2 \text { weeks in the doctor's office }\end{array}$ & \\
\hline & $\begin{array}{l}\text { IV for } 30 \text { minutes every } 3 \text { weeks in the } \\
\text { doctor's office }\end{array}$ & \\
\hline & $\begin{array}{c}2 \text { pills twice a day, plus } 1 \text { additional pill } \\
\text { once a day at home }\end{array}$ & \\
\hline
\end{tabular}

Figure 1. Example choice questions. (A) Example discrete-choice experiment question. (B) Example best-worst scaling question.

IV: Intravenous. 
preferences in attribute levels across alternatives in each choice question and corrects for potential estimation bias [43,44]. The preference weights derived from the RPL analysis are a relative measure of the strength of preferences. More-preferred outcomes have higher preference weights, and a broader spread of preference weights within each attribute measure indicates stronger preferences for higher valued levels over lower valued levels.

Months of PFS, risk of colitis, risk of hormone gland problems and risk of extreme sensitivity to the sun were modeled as continuous linear variables after specification tests corroborated that the marginal effects of these attributes could be assumed to be constant within the range of levels shown to respondents. In other words, one-unit increases in the attributes were valued equally by respondents, regardless of the starting point for the increase. Mode of administration and fever were modeled as effects-coded variables. With effects coding, zero indicates the mean effect across all attribute levels rather than the omitted level, as in dummy coding [45]. Wald tests were used to identify differences between levels for each attribute at the 95\% CI.

Treatment choices among patients who reported an initial diagnosis of stage IV cancer versus those with an earlier disease stage at initial diagnosis were analyzed using an RPL model with the same specification as the full-sample model. We created a dummy-coded variable that was equal to 1 if the respondent had stage IV cancer at diagnosis and interacted the dummy variable with each of the explanatory variables used in the main model. Each interaction variable was the product of the dummy-coded variable and one of the treatment attribute variables. The parameter on each of these interaction terms could be interpreted as the difference between the two subgroups for the preference weights for the corresponding attribute level. Differences in preferences were tested through a joint test of the significance of all the interaction terms (Wald test).

\section{Preference shares}

Results from the RPL model can be used to predict the probability that the average respondent would select one treatment profile over another. To calculate the probability that a respondent would select each profile if offered a choice between the two, we calculated a net clinical benefit score for each medication as the weighted sum of each medication profile's attribute levels, where the weights are the preference weights from the RPL model for the corresponding attribute level in the medication profile.

We used the results to explore preferences for treatments with typical AEs associated with different targeted therapy and immunotherapy options, assuming equal efficacy (holding PFS constant). We simulated a choice scenario comparing a targeted therapy profile that included a higher risk of fever, a higher risk of extreme sensitivity to the sun, and pills as the mode of administration with an immunotherapy profile that included risks of colitis and hormone gland problems, and administration by IV infusion for $2.5 \mathrm{~h}$ every 3 weeks in the doctor's office for four doses, then IV infusion for 60 min every 2 weeks. The coefficient for the higher risk of fever in the targeted therapy profile was calculated using a weighted average of the three levels of fever presented in the DCE questions and based on clinical data from the combination of dabrafenib and trametinib $[21,26]$. The coefficient for the risk of extreme sun sensitivity profile was based on clinical data from the combination of vemurafenib and cobimetinib [27]. The risk of colitis and hormone gland problems (hypothyroidism) for the immunotherapy profile were based on the treatment-related, all grades percentage risk for Opdivo (nivolumab) and Yervoy (ipilimumab) combination [31]. The preference share profiles and weighted average percentages used for the composite fever attribute are presented in Table 2.

\section{Best-worst scaling}

Importance weights were estimated using an RPL model that related respondents' choices for the most- and leastpreferred items to the item-specific variables [36]. Larger coefficients indicate that the item was most preferred. Conversely, smaller coefficients indicate that the item was less preferred. Relative importance weights were created from the log-odds importance weights using a probability-based rescaling procedure [46].

\section{Results}

Study sample

Global Perspectives invited 23,157 individuals by e-mail to be screened for study eligibility. Of those invited, 455 individuals responded. Approximately half of these individuals (207) were eligible and consented to participate, and $200(96.6 \%)$ completed the survey, which took respondents an average of $18.6 \mathrm{~min}$ to complete. Self-reported demographic and clinical characteristics of the respondents are shown in Table 3. The mean age of patients was 47 years (range: $20-76$ ), and 111 (55.5\%) were men. The stage of melanoma reported most commonly at diagnosis 
Table 2. Preference share profiles for targeted therapy and immunotherapy, assuming same efficacy.

\begin{tabular}{|c|c|c|}
\hline Attribute & Targeted therapy & Immunotherapy \\
\hline $\begin{array}{l}\text { How long the medicine will keep the cancer stable } \\
\text { (progression-free survival) }\end{array}$ & 12 months & 12 months \\
\hline How you take the medicine & Pills taken twice-a-day at home & $\begin{array}{l}\text { IV infusion for } 2.5 \mathrm{~h} \text { every } 3 \text { weeks in the doctor's office } \\
\text { for } 4 \text { doses, then IV infusion for } 60 \text { min every } 2 \text { weeks }\end{array}$ \\
\hline Risk of tears or holes in your intestines (colitis) ${ }^{\dagger}$ & $0 \%$ & $13 \%$ \\
\hline Risk of hormone gland problems & $0 \%$ & $17 \%$ \\
\hline Fever for a few days each month $\S$ & $29 \%$ none, $66 \%$ mild-to-moderate, $5 \%$ severe & None \\
\hline Risk of extreme sensitivity to the sun $\mathbb{I}$ & $2.36 \%$ & $0 \%$ \\
\hline \multicolumn{3}{|c|}{$\begin{array}{l}\dagger \text { For the immunotherapy profile, the risk of colitis is based on the treatment related, all grades percentage risk for Opdivo (nivolumab) and Yervoy (ipilimumab) combination [31]. } \\
\text { ‡For the immunotherapy profile, the risk of hormone gland problems is based on hypothyroidism treatment related, all grades percentage risk for Opdivo (nivolumab) and Yervoy } \\
\text { (ipilimumab) combination [31]. } \\
\S_{\text {For the fever attribute in the targeted therapy profile, a weighted average of the fever levels presented in the DCE questions was constructed by multiplying the preference weights for }} \\
\text { the three levels of the fever attribute by the probability of fever in Robert et al. [21]. For the survey, the description of mild-to-moderate fever corresponded to grade } 1 \text { or } 2 \text { and severe fever } \\
\text { corresponded to grade } 3 \text { or } 4 \text {. The weighted average was derived by the following equation: FEV } 1 * 0.29+\mathrm{FEV} 2^{\star} 0.66+\mathrm{FEV} 3 * 0.05=0.97 * 0.29+0.19 * 0.66+(-1.16)^{\star} 0.05=0.35 \text {. } \\
\mathbb{I} \text { For the targeted therapy, the risk of extreme sun sensitivity is based on the risk of grade } 3 \text { sun sensitivity in Larkin et al. [27]. } \\
\text { DCE: Discrete-choice experiment. }\end{array}$} \\
\hline
\end{tabular}

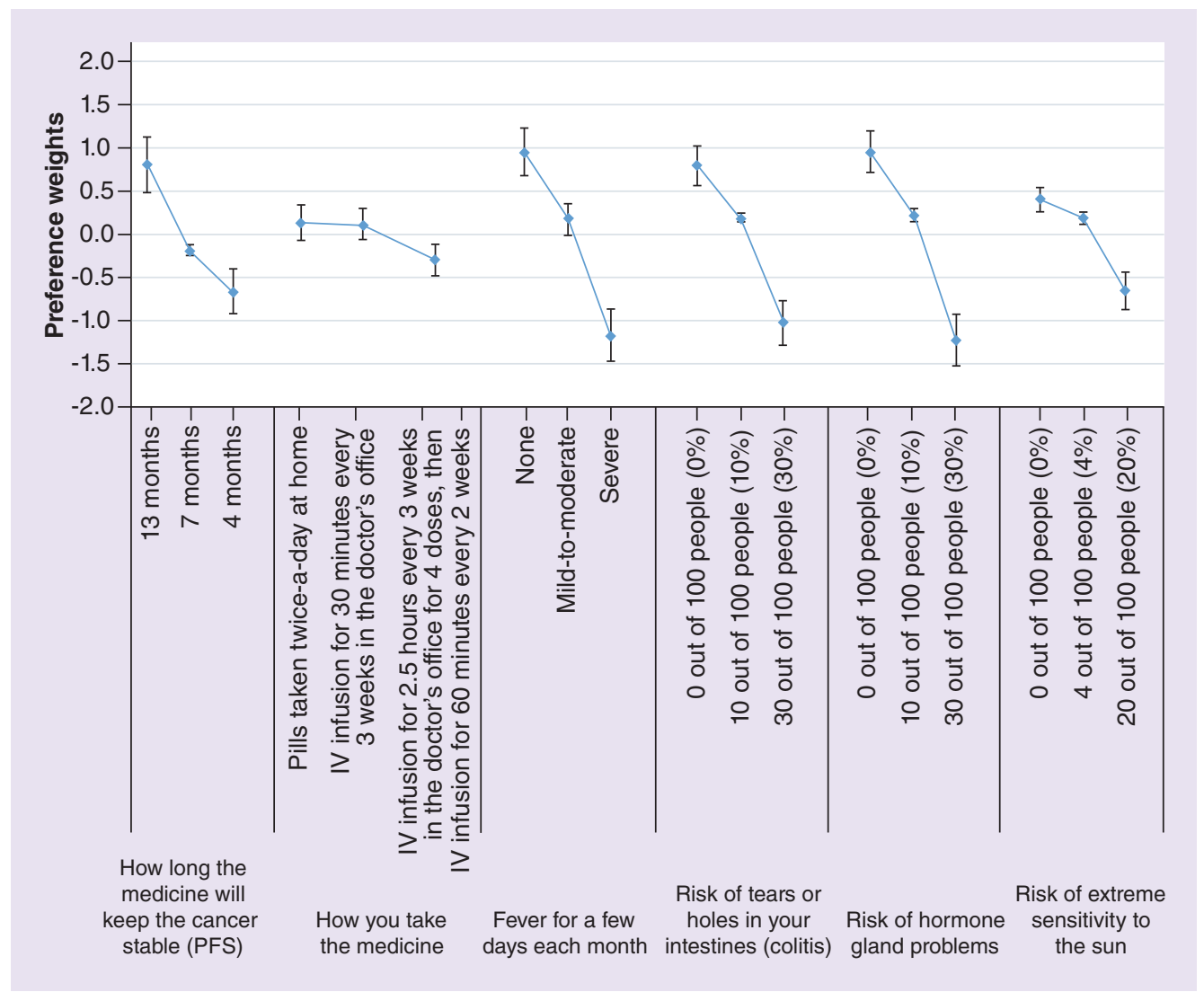

Figure 2. Preference weights for each attribute level. The vertical bars around each mean preference weight represent the $95 \%$ Cls about the point estimate.

IV: Intravenous; PFS: Progression-free survival.

was stage III $(\mathrm{n}=76[38.0 \%])$ or stage IV $(\mathrm{n}=85[42.5 \%])$, and more than half of patients had been diagnosed at least 2 years prior to completing the survey.

\section{Preference weights}

Figure 2 presents the mean preference weight values for each attribute level. Overall, the preference weights were ordered as expected: respondents preferred longer PFS, no fever, no chance of colitis, no chance of hormone gland 
Table 3. Patient characteristics.

Demographic characteristic

Age (years)

What is your gender?

What is the highest level of education you have completed?

Which of the following best describes your employment status?

\begin{tabular}{|l}
\hline \\
\hline \\
\hline \\
\hline
\end{tabular}

What type of health insurance do you have? (check all that apply)

\section{Disease characteristic}

When cancer is diagnosed, doctors will often describe the 'stage' of the cancer. The descriptions below match different stages of

melanoma. Please pick the description below that best describes your

category that describes the most severe diagnosis you received

\begin{tabular}{|c|c|c|}
\hline & Stage $1^{\dagger}$ & $2(1.0 \%)$ \\
\hline & Stage $2^{\dagger}$ & $36(18.0 \%)$ \\
\hline & Stage 3 & $76(38.0 \%)$ \\
\hline & Stage 4 & $85(42.5 \%)$ \\
\hline \multirow[t]{6}{*}{ Time since diagnosis } & Less than 6 months & $5(2.5 \%)$ \\
\hline & 6 months to less than 1 year & $4(2.0 \%)$ \\
\hline & 1 year to less than 2 years & $41(20.5 \%)$ \\
\hline & 2 years to less than 5 years & $90(45.0 \%)$ \\
\hline & 5 years to less than 10 years & $46(23.0 \%)$ \\
\hline & 10 years or more & $14(7.0 \%)$ \\
\hline \multirow{6}{*}{$\begin{array}{l}\text { What types of treatments or medicines are you currently using to treat } \\
\text { the melanoma, other than surgery? (Please check all that apply) } \\
(\mathrm{N}=157)\end{array}$} & Targeted therapy & $63(40.1 \%)$ \\
\hline & Immunotherapy & $93(59.2 \%)$ \\
\hline & Chemotherapy & $93(59.2 \%)$ \\
\hline & Radiation therapy & $107(68.2 \%)$ \\
\hline & Other & $3(1.9 \%)$ \\
\hline & Do not know/not sure of type of treatment & $1(0.6 \%)$ \\
\hline
\end{tabular}

Statistic or category

Mean (SD)

Minimum, maximum

Female

Male

High school or equivalent (e.g., GED)

Some college but no degree

Technical school

Associate's degree (2-year college degree)

4-year college degree (e.g., BA, BS)

Some graduate school but no degree

Graduate or professional degree (e.g., MBA, MS, MD, PhD)

Employed full-time

Employed part-time

Self-employed

Homemaker

Retired

Disabled/unable to work

Unemployed

I do not have health insurance

Private insurance that I pay for myself

Private insurance that my or my spouse's employer pays all or part of

Medicaid

Medicare

Veterans' health insurance

Other

Stage $0^{\dagger}$

$1(0.5 \%)$

$19(9.5 \%)$

$2(1.0 \%)$

$4(2.0 \%)$

$87(43.5 \%)$

$48(24.0 \%)$

$56(28.0 \%)$

$108(54.0 \%)$

$4(2.0 \%)$

$4(2.0 \%)$

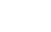

\begin{tabular}{|l|}
\hline What type of health insurance do you have? (check all that apply) \\
\hline \\
\hline \\
\hline
\end{tabular}

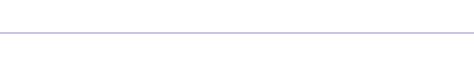


Table 3. Patient characteristics (cont.).

\begin{tabular}{|c|c|c|}
\hline $\begin{array}{l}\text { Demographic characteristic } \\
\text { What types of treatments or medicines have you used in the past to } \\
\text { treat the melanoma (before your current treatment, if you are taking } \\
\text { one)? (Please check all that apply; } N=186 \text { ) }\end{array}$ & \multirow{2}{*}{$\begin{array}{l}\text { Statistic or category } \\
\text { Targeted therapy }\end{array}$} & \multirow{2}{*}{$\begin{array}{l}\text { Overall }(\mathrm{N}=200) \\
42(22.6 \%)\end{array}$} \\
\hline \multirow{7}{*}{$\begin{array}{l}\text { What types of treatments or medicines have you used in the past to } \\
\text { treat the melanoma (before your current treatment, if you are taking } \\
\text { one)? (Please check all that apply; } N=186 \text { ) }\end{array}$} & & \\
\hline & Immunotherapy & $142(76.3 \%)$ \\
\hline & Chemotherapy & $116(62.4 \%)$ \\
\hline & Radiation therapy & $130(69.9 \%)$ \\
\hline & Other & $7(3.8 \%)$ \\
\hline & Do not know/not sure of type of treatment & 0 \\
\hline & None & 0 \\
\hline
\end{tabular}

problems and no chance of extreme sensitivity to the sun. Statistical analysis of respondent choices within each attribute revealed that preferences for each attribute level were significantly different from each other, except pills taken twice-a-day at home and IV infusion for 30 min every 3 weeks in the doctor's office. Respondents were indifferent between these two levels. When comparing the AEs presented in the study, respondents indicated that avoiding a 30\% risk of colitis or hormone gland problems and avoiding a severe fever were more important than avoiding a $20 \%$ risk of extreme sun sensitivity ( $\mathrm{p}<0.01)$.

In the subgroup analysis, respondents with an initial diagnosis of stage IV melanoma had significantly different preferences from respondents initially diagnosed with an earlier stage $(\mathrm{p}<0.01)$. Respondents diagnosed at stage IV placed relatively more weight on longer PFS and reducing the risks of colitis and hormone gland problems than respondents with an earlier disease stage at initial diagnosis (Figure 3). Patients aged 47 years and older also had significantly different preferences than younger patients $(\mathrm{p}<0.05)$. Older respondents placed more emphasis on longer PFS than younger respondents, and they placed similar value on the increases in PFS and reductions the risk of colitis and hormone problems presented. Respondents younger than 47 years focused on avoiding treatment risks and were insensitive to the changes in PFS offered (Figure 4).

\section{Preference shares}

Approximately $85 \%$ of respondents preferred a treatment profile with AEs and mode of administration similar to a targeted therapy when compared with a treatment profile with AEs and mode of administration similar to an immunotherapy, based on the assumptions in Table 2 (Figure 5).

\section{Best-worst scaling}

Model results

To further assess patients' preferences for mode of administration, we conducted a best-worst scaling experiment in which patients chose from multiple dosing options. In general, patients preferred oral dosing to an IV infusion. However, many respondents preferred a less burdensome IV infusion schedule to a complex dosing schedule that includes multiple pills several times a day in a regimen that includes drug holidays (Figure 6). Based on the CIs, the importance weights for the two most-preferred modes of administration, 'one pill once-a-day at home' and 'two pills twice-a-day at home', were not statistically significantly different from one another, nor were the three least-preferred items. The frequency and percentage of each item that was chosen as least and most preferred are presented in Supplementary Table 1.

\section{Discussion}

Prior to last decade, treatment options for patients diagnosed with advanced melanoma provided limited clinical benefit. The introduction of immune checkpoint inhibitors and molecularly targeted treatments offers physicians and their patients more choices and, for some patients, improved PFS and OS; however, each new therapy also presents a constellation of potential benefits and risks that must be weighed. In addition, the dosing method and frequency varies considerably among treatments.

We quantified patients' preferences for treatment attributes and modes of administration found in commercially available treatments for metastatic melanoma. The range of PFS reported in clinical data for targeted and im- 


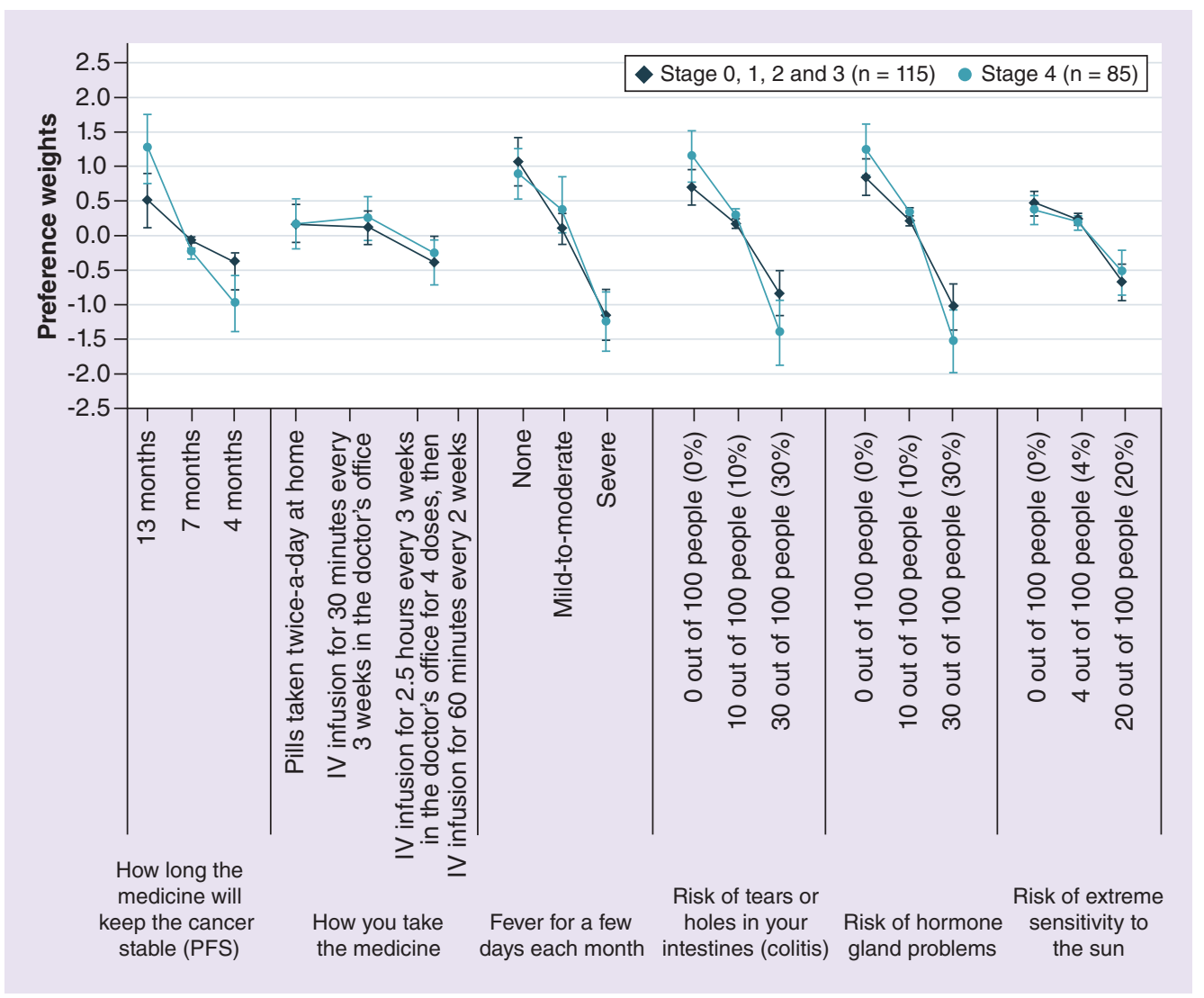

Figure 3. Subgroup preference weights by stage at initial diagnosis. The vertical bars around each mean preference weight represent the $95 \% \mathrm{Cls}$ about the point estimate.

IV: Intravenous; PFS: Progression-free survival.

munotherapies varies from about 4 to 13 months [5,8]. Our findings suggest that respondents value an increase in PFS from 4 to 13 months and are willing to trade the risk of AEs such as hormone gland problems, colitis and severe fever for a longer PFS benefit. In addition, patients with stage IV melanoma at diagnosis placed greater weight on increasing PFS and on lowering the risk of colitis and hormone gland problems than patients with an earlier stage of disease at diagnosis. Patients aged 47 years or older placed more emphasis on longer PFS than younger respondents, whereas younger respondents focused on avoiding treatment risks and were insensitive to changes in PFS.

Some monotherapies are administered as a continuous fixed regimen of a single pill once-a-day or two pills twicea-day. Targeted therapy combinations typically range from twice-a-day oral administration to more complicated oral regimens, including those with a drug holiday (3 weeks on, 1 week off). Immune checkpoint inhibitors are typically administered as IV infusions with varying schedules. Our study respondents overall preferred taking pills to receiving IV administration; but, they preferred a less burdensome IV schedule to taking multiple pills several times a day (pill burden of $\geq 8$ per day) or complicated dosing schedules.

Calculations of the probability that respondents would select therapies with characteristics (AEs and mode of administration) common to either targeted therapies or immunotherapies suggest that, on average, respondents preferred targeted therapy over immunotherapy treatments. The risks from fever and extreme sensitivity to the sun taken from clinical data on targeted therapies were preferred to the risks of colitis and hormone gland problems at the levels observed in data on immunotherapies.

The study has some limitations that should be considered when assessing the results. The diagnosis of advanced or metastatic melanoma was self-reported and the BRAFV600 status of respondents was not assessed. Although some respondents were recruited through patient organizations and proprietary databases that screen respondents, other respondents were not independently screened. Patient-friendly descriptions of the attributes were presented, 


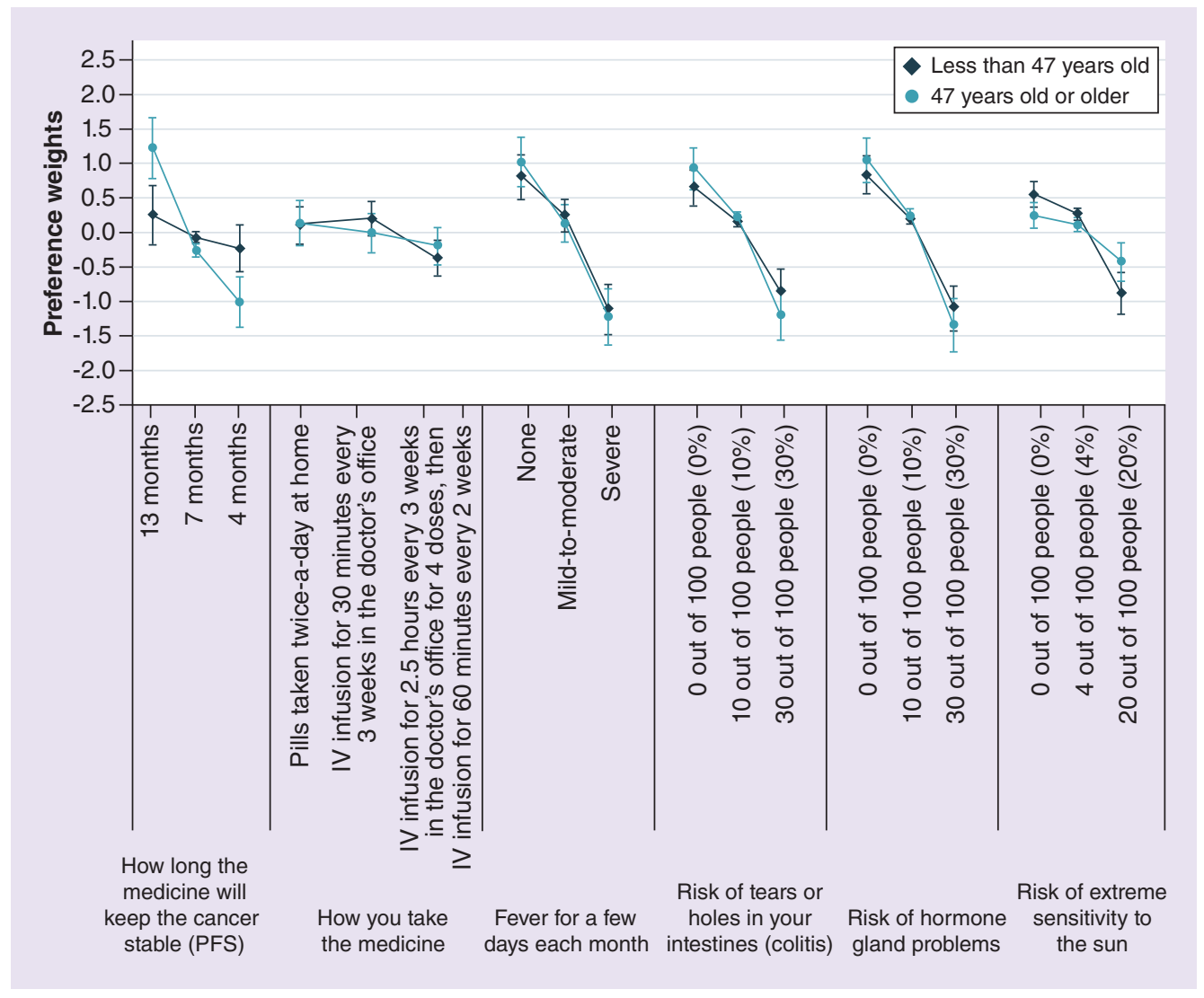

Figure 4. Subgroup preference weights by age. The vertical bars around each mean preference weight represent the $95 \% \mathrm{Cls}$ about the point estimate.

IV: Intravenous; PFS: Progression-free survival.

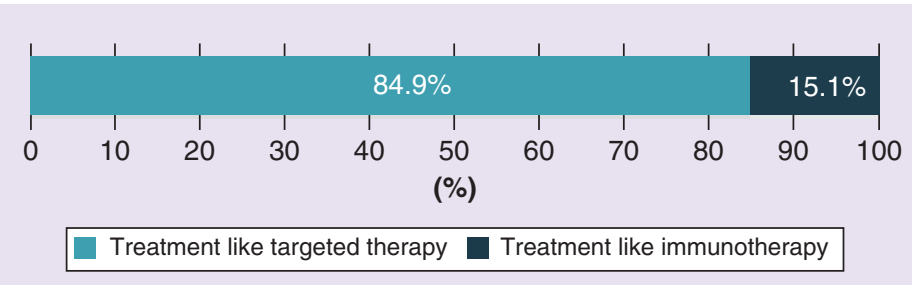

Figure 5. Preference shares for targeted therapy and immunotherapy with typical adverse events and modes of administration, assuming equal progression-free survival. The figure presents the percentage of respondents who prefer a treatment with an adverse event profile and mode of administration like those of targeted therapy versus the percentage of respondents who prefer a treatment with an adverse event profile and mode of administration like those of immunotherapy, assuming equal progression-free survival.

but not all patients may fully understand complex AEs such as endocrinopathies based on the description provided in the survey.

The attributes and levels were based on data from clinical trials for the therapies considered, but the sample was not selected to match the samples for the clinical trials. The sample may not match a nationally representative sample of patients with metastatic melanoma, so the preferences may not be generalizable to all patients.

The survey presents hypothetical scenarios to respondents. Decisions made in the survey may not fully predict decisions made in a clinical setting, where other considerations such as the physician's preferences, attributes such as duration of treatment and other AEs not included in the DCE may come into play. In addition, the final survey was administered online. The method of survey administration can influence respondents' responses, although 


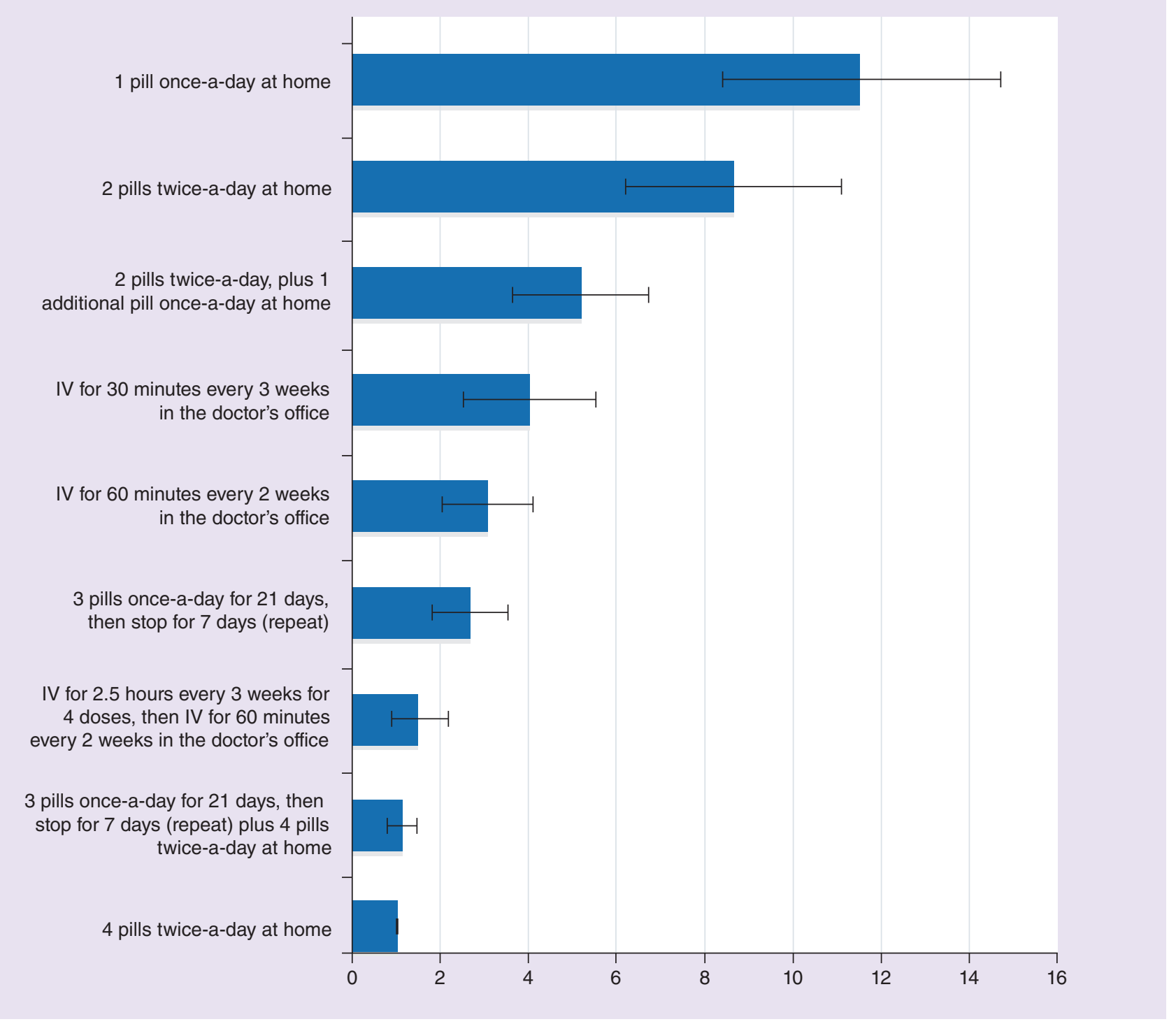

Figure 6. Best-worst scaling relative preference weights. The horizontal bars around each mean preference weight represent the $95 \%$ Cls about the point estimate.

IV: Intravenous.

previous research has shown in which direction responses might change and how to optimize the mode of survey administration for DCE surveys [47]. Some research has found that the results from online stated-preference surveys are not statistically different from those elicited through face-to-face interviews [48,49].

The PFS attribute was chosen due to the availability of patient data to support the attribute levels. Targeted agents have been associated with longer PFS $[1,2,8,50]$, although comparable efficacy has been observed with a combination of immune checkpoint inhibitors [31]. At the time of the survey's design, data on OS were not available for every treatment. Therefore, conclusions are limited to patient choice based on PFS levels. The results may have differed if OS were included as an attribute. The preference share calculations held efficacy (PFS) constant and did not include the full range of administration modes for targeted and immunotherapies.

\section{Conclusion}

In this study, patients had distinct preferences for changes in attributes presented in the DCE, including PFS, the $\mathrm{AE}$ risks and the different modes and schedules of administration. Overall, patients preferred a treatment profile that included AEs and mode of administration associated with targeted therapies over immunotherapies. Taking 
patient preferences into account may help patients find a treatment that fits their circumstances and tolerance for different types of AEs. A careful discussion of AEs may also help patients anticipate and manage AEs, leading to higher treatment satisfaction. Addressing patient preferences may help patients obtain the full benefit from the growing number of therapeutic options available for advanced and metastatic melanoma.

\section{Summary points}

- Recent advances in the treatment of metastatic melanoma, including the introduction of immune checkpoint inhibitors and molecularly targeted treatments, have resulted in more treatment choices and improved outcomes for many patients. Each of the available treatments for metastatic melanoma carries potential benefits and risks that must be weighed.

- The aim of this study was to investigate patient preferences for the clinical attributes that differ among first-line treatments for BRAFV600-positive metastatic melanoma.

- A discrete-choice experiment was conducted to elicit patients' relative preferences for treatment attributes including progression-free survival, mode and frequency of administration, and treatment-related adverse events.

- Analysis of the discrete-choice experiment data using a random-parameters logit model revealed distinct preferences among 200 survey respondents. Avoiding a 30\% risk of colitis or hormone gland problems and avoiding severe fever were more important to patients than avoiding a $20 \%$ risk of extreme sun sensitivity ( $p$ $<0.05)$.

- A best-worst scaling exercise evaluating a range of treatment modes and administration schedules revealed that patients prefer taking pills at home rather than receiving intravenous infusions in a doctor's office, but they preferred a less burdensome intravenous schedule to a complicated pill dosing schedule.

- When attributes were combined in an analysis of preference, approximately $85 \%$ of respondents preferred the risk profile and mode of administration similar to a targeted therapy over a profile similar to an immunotherapy, holding efficacy constant.

- Overall, respondents in this study preferred a treatment profile that included adverse events and mode of administration associated with targeted therapies over immunotherapies.

\section{Supplementary data}

To view the supplementary data that accompany this paper please visit the journal website at: www.futuremedicine.com/doi/suppl/10.2217/fon-2018-0871

\section{Financial \& competing interests disclosure}

Financial support for the study was provided by Novartis Pharmaceutical Corporation pursuant to a research contract between Novartis and RTI Health Solutions. At the time the study was conducted, C Mansfield and K Gallaher were full time employees of RTI Health Solutions (RTI-HS), which received research funding from Novartis Pharmaceutical Corporation to conduct the study. No payments for the study were made to the RTI-HS authors. B Ndife, J Chen and S Ghate were employees of Novartis at the time of the study. The authors have no other relevant affiliations or financial involvement with any organization or entity with a financial interest in or financial conflict with the subject matter or materials discussed in the manuscript apart from those disclosed.

Medical writing support was provided by K Hede of RTI-HS and was funded by Novartis.

Ethical conduct of research

The authors state that they obtained appropriate institutional review board approval and followed the principles outlined in the Declaration of Helsinki for this study. Informed consent was obtained from the survey respondents.

\section{Open access}

This work is licensed under the Attribution-NonCommercial-NoDerivatives 4.0 Unported License. To view a copy of this license, visit http://creativecommons.org/licenses/by-nc-nd/4.0/

\section{References}

Papers of special note have been highlighted as: $\bullet$ of interest; $\bullet \bullet$ of considerable interest

1. Devji T, Levine O, Neupane B, Beyene J, Xie F. Systemic therapy for previously untreated advanced BRAF-mutated melanoma: a systematic review and network meta-analysis of randomized clinical trials. JAMA Oncol. 3(3), 366-373 (2017).

2. Johnson DB, Pollack MH, Sosman JA. Emerging targeted therapies for melanoma. Expert Opin. Emerg. Drugs 21(2), 195-207 (2016). 
3. Karachaliou N, Gonzalez-Cao M, Sosa A et al. The combination of checkpoint immunotherapy and targeted therapy in cancer. Ann. Transl. Med. 5(19), 388 (2017).

4. Kelley MC. Immune responses to BRAF-targeted therapy in melanoma: is targeted therapy immunotherapy? Crit. Rev. Oncog. 21(1-2), 83-91 (2016).

5. Luke JJ, Flaherty KT, Ribas A, Long GV. Targeted agents and immunotherapies: optimizing outcomes in melanoma. Nat. Rev. Clin. Oncol. 14(8), 463-482 (2017).

6. Margolin K. The promise of molecularly targeted and immunotherapy for advanced melanoma. Curr. Treat. Options Oncol. 17(9), 48 (2016).

7. Silva IP, Long GV. Systemic therapy in advanced melanoma: integrating targeted therapy and immunotherapy into clinical practice. Curr. Opin. Oncol. 29(6), 484-492 (2017).

8. Ugurel S, Rohmel J, Ascierto PA et al. Survival of patients with advanced metastatic melanoma: the impact of novel therapies-update 2017. Eur. J. Cancer 83, 247-257 (2017).

9. Volpe VO, Klufas DM, Hegde U, Grant-Kels JM. The new paradigm of systemic therapies for metastatic melanoma. J. Am. Acad. Dermatol. 77(2), 356-368 (2017).

10. Gilligan T, Coyle N, Frankel RM et al. Patient-clinician communication: American Society of Clinical Oncology Consensus Guideline. J. Clin. Oncol. 35(31), 3618-3632 (2017).

-• Describes the importance of shared decision-making between physicians and patients.

11. US FDA. Patient preference information - voluntary submission, review in premarket approval applications, humanitarian device exemption applications, and de novo requests, and inclusion in decision summaries and device labeling: guidance for industry, food and drug administration staff, and other stakeholders. www.fda.gov/downloads/MedicalDevices/DeviceRegulationandGuidance/GuidanceDocuments/UCM446680.pdf

- Describes the role of patient preferences in healthcare decision making.

12. US FDA. Enhancing benefit-risk assessment in regulatory decision-making. www.fda.gov/ForIndustry/UserFees/PrescriptionDrugUserFee/ucm326192.htm

13. Hauber AB, González JM, Groothuis-Oudshoorn CGM et al. Statistical methods for the analysis of discrete choice experiments: a report of the ISPOR conjoint analysis good research practices task force. Value Health 19(4), 300-315 (2016).

- Describes the best practices for analyzing discrete-choice experiment data.

14. Ho MP, Gonzalez JM, Lerner HP et al. Incorporating patient-preference evidence into regulatory decision making. Surg. Endosc. 29(10), 2984-2993 (2015).

15. Goossens LMA, Rutten-van Molken M, Boland MRS et al. ABC index: quantifying experienced burden of COPD in a discrete choice experiment and predicting costs. BMJ Open 7(12), e017831 (2017).

16. Liu FX, Witt EA, Ebbinghaus S, DiBonaventura Beyer G, Basurto E, Joseph RW. Patient and oncology nurse preferences for the treatment options in advanced melanoma: a discrete choice experiment. Cancer Nurs. 42(1), e52-e59 (2019).

-• Previous study conducted to evaluate patients' and oncology nurses' preferences for advanced melanoma treatments.

17. Liu FX, Witt EA, Ebbinghaus $S$ et al. Patient and oncologist preferences for attributes of treatments in advanced melanoma: a discrete choice experiment. Patient Prefer. Adherence 11, 1389-1399 (2017).

-• Previous study conducted to evaluate patients' and oncologists' preferences for advanced melanoma treatments.

18. Hermel DJ, Ott PA. Combining forces: the promise and peril of synergistic immune checkpoint blockade and targeted therapy in metastatic melanoma. Cancer Metastasis Rev. 36(1), 43-50 (2017).

19. Reed Johnson F, Lancsar E, Marshall D et al. Constructing experimental designs for discrete-choice experiments: report of the ISPOR Conjoint Analysis Experimental Design Good Research Practices Task Force. Value Health 16(1), 3-13 (2013).

20. Bridges JF, Hauber AB, Marshall D et al. Conjoint analysis applications in health - a checklist: a report of the ISPOR Good Research Practices for Conjoint Analysis Task Force. Value Health 14(4), 403-413 (2011).

- Describes best practices for conducting discrete-choice experiment studies.

21. Robert C, Karaszewska B, Schachter J et al. Improved overall survival in melanoma with combined dabrafenib and trametinib. N. Engl. J. Med. 372(1), 30-39 (2015).

22. Robert C, Karaszewska B, Schachter J et al. Two-year estimate of overall survival in COMBI-v, a randomized, open-label, Phase III study comparing the combination of dabrafenib and trametinib with vemurafenib as first-line therapy in patients with unresectable or metastatic BRAF V600E/K mutation-positive cutaneous melanoma. Eur. J. Cancer 51(Suppl. 3), S663, Abstract 3301 (2015).

23. Robert C, Karaszewska B, Schachter J et al. Three-year estimate of overall survival in COMBI-v, a randomized Phase III study evaluating first-line debrafenib + trametinib in patients with unresectable or metastatic BRAF V600E/K-mutant cutaneous melanoma. Ann. Oncol. 27(Suppl. 6), LBA40 (2016).

24. Robert C, Schachter J, Long GV et al. Pembrolizumab versus ipilimumab in advanced melanoma. N. Engl. J. Med. 372(26), 2521-2532 (2015). 
25. Long GV, Flaherty KT, Stroyakovskiy D et al. Dabrafenib plus trametinib versus dabrafenib monotherapy in patients with metastatic BRAF V600E/K-mutant melanoma: long-term survival and safety analysis of a Phase 3 study. Ann. Oncol. 28(7), 1631-1639 (2017).

26. Long GV, Stroyakovskiy D, Gogas $\mathrm{H}$ et al. Dabrafenib and trametinib versus dabrafenib and placebo for Val600 BRAF-mutant melanoma: a multicentre, double-blind, Phase III randomised controlled trial. Lancet 386(9992), 444-451 (2015).

27. Larkin J, Ascierto PA, Dreno B et al. Combined vemurafenib and cobimetinib in BRAF-mutated melanoma. N. Engl. J. Med. 371(20), 1867-1876 (2014).

28. Ascierto PA, McArthur GA, Dreno B et al. Cobimetinib combined with vemurafenib in advanced BRAF(V600)-mutant melanoma (coBRIM): updated efficacy results from a randomised, double-blind, Phase III trial. Lancet Oncol. 17(9), 1248-1260 (2016).

29. Schachter J, Ribas A, Long GV et al. Pembrolizumab versus ipilimumab for advanced melanoma: final overall survival results of a multicentre, randomised, open-label Phase III study (KEYNOTE-006). Lancet 390(10105), 1853-1862 (2017).

30. Larkin J, Chiarion-Sileni V, Gonzalez R et al. Combined nivolumab and ipilimumab or monotherapy in untreated melanoma. N. Engl. J. Med. 373(1), 23-34 (2015).

31. Wolchok JD, Chiarion-Sileni V, Gonzalez R et al. Overall survival with combined nivolumab and ipilimumab in advanced melanoma. N. Engl. J. Med. 377(14), 1345-1356 (2017).

32. Kuhfeld W. Efficient experimental designs using computerized searches. Presented at: Sawtooth Software Conference Proceedings. Seattle, WA, USA, August 1997.

33. Kuhfeld W. Marketing research methods in SAS: experimental design, choice, conjoint, and graphical techniques. SAS Institute, Inc., NC, USA. (2010). http://support.sas.com/techsup/technote/mr2010.pdf

34. Kuhfeld W, Tobias F, Garratt M. Efficient experimental design with marketing research applications. J. Mark. Res. 31, 545-557 (1994).

35. Chrzan K, Orme B. An overview and comparison of design strategies for choice-based conjoint analysis (2000). www.sawtoothsoftware.com/download/techpap/desgncbc.pdf

36. Yuan Z, Levitan B, Burton P, Poulos C, Brett Hauber A, Berlin JA. Relative importance of benefits and risks associated with antithrombotic therapies for acute coronary syndrome: patient and physician perspectives. Curr. Med. Res. Opin. 30(9), 1733-1741 (2014).

37. Louviere J, Flynn TF, Marley AA. Best-Worst Scaling Theory, Methods and Applications. Cambridge University Press, Cambridge, UK (2015).

38. Flynn TN, Louviere JJ, Peters TJ, Coast J. Best-worst scaling: what it can do for health care research and how to do it. J. Health Econ. 26(1), 171-189 (2007).

39. Hauber AB, Mohamed AF, Johnson FR et al. Understanding the relative importance of preserving functional abilities in Alzheimer's disease in the United States and Germany. Qual. Life Res. 23(6), 1813-1821 (2014).

40. Ross M, Bridges JF, Ng X et al. A best-worst scaling experiment to prioritize caregiver concerns about ADHD medication for children. Psychiatr. Serv. 66(2), 208-211 (2015).

41. Peay HL, Hollin I, Fischer R, Bridges JF. A community-engaged approach to quantifying caregiver preferences for the benefits and risks of emerging therapies for Duchenne muscular dystrophy. Clin. Ther. 36(5), 624-637 (2014).

42. Ungar WJ, Hadioonzadeh A, Najafzadeh M, Tsao NW, Dell S, Lynd LD. Quantifying preferences for asthma control in parents and adolescents using best-worst scaling. Respir. Med. 108(6), 842-851 (2014).

43. Train K. Discrete Choice Methods with Simulation. Cambridge University Press, Cambridge, UK, doi:https://doi.org/10.1017/CBO9780511805271 (2009).

44. Train K, Sonnier G. Mixed logit with bounded distributions of correlated partworths. In: Applications of Simulation Methods in Environmental and Resource Economics. R Scarpa, A Alberini (Eds). Springer, Dordrecht, The Netherlands (2005).

45. Hensher DA, Rose JM, Greene WH. Applied Choice Analysis. Cambridge University Press, Cambridge, UK (2005).

46. The MaxDiff System Technical Paper (2013). www.sawtoothsoftware.com/download/techpap/maxdifftech.pdf

47. Leggett CG, Kleckner NS, Boyle K, Dufield JW, Mitchell RC. Social desirability bias in contingent valuation surveys administered through in-person interviews. Land Economics 79(4), 561-575 (2003).

48. Nielsen JS. Use of the internet for willingness-to-pay surveys: a comparison of face-to-face and web-based interviews. Resour. Energy Econ. 33(1), 119-129 (2011).

49. Marta-Pedrosa C, Freitas H, Domingos T. Testing for the survey mode effect on contingent valuation data quality: a case study of web based versus in-person interviews. Ecol. Econ. 62(3-4), 388-398 (2007).

50. Heppt MV, Dietrich C, Graf SA, Ruzicka T, Tietze JK, Berking C. The systemic management of advanced melanoma in 2016. Oncol. Res. Treat. 39(10), 635-642 (2016). 